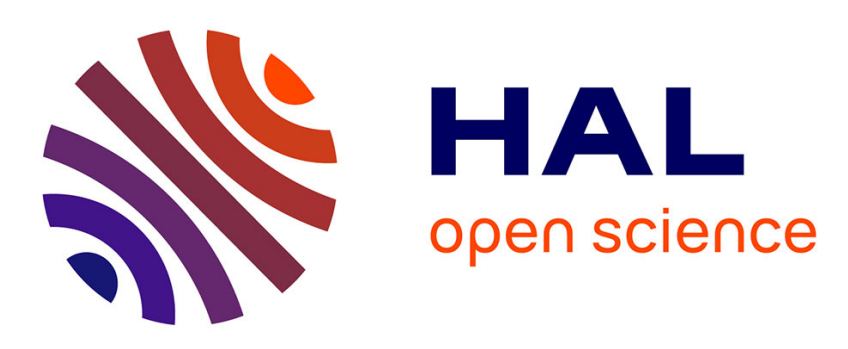

\title{
Thick sets, multiple-valued mappings, and possibility theory
}

\author{
Didier Dubois, Luc Jaulin, Henri Prade
}

\section{To cite this version:}

Didier Dubois, Luc Jaulin, Henri Prade. Thick sets, multiple-valued mappings, and possibility theory. Vladik Kreinovich. Statistical and Fuzzy Approaches to Data Processing, with Applications to Econometrics and Other Areas, 892, Springer, pp.101-109, 2020, Studies in Computational Intelligence, 978-3-030-45618-4. 10.1007/978-3-030-45619-1_8. hal-02924428

\section{HAL Id: hal-02924428 \\ https://hal-ensta-bretagne.archives-ouvertes.fr/hal-02924428}

Submitted on 3 May 2021

HAL is a multi-disciplinary open access archive for the deposit and dissemination of scientific research documents, whether they are published or not. The documents may come from teaching and research institutions in France or abroad, or from public or private research centers.
L'archive ouverte pluridisciplinaire HAL, est destinée au dépôt et à la diffusion de documents scientifiques de niveau recherche, publiés ou non, émanant des établissements d'enseignement et de recherche français ou étrangers, des laboratoires publics ou privés. 


\title{
Thick sets, multiple-valued mappings, and possibility theory
}

\author{
Didier Dubois ${ }^{1}$, Luc Jaulin ${ }^{2}$, Henri Prade ${ }^{1}$ \\ ${ }^{1}$ IRIT - CNRS, 118, route de Narbonne, 31062 Toulouse Cedex 09, France \\ \{dubois, prade\}@irit.fr \\ ${ }^{2}$ Lab-STICC, ENSTA-Bretagne, 2 rue François Verny, 29200 Brest, France, \\ lucjaulin@gmail.com
}

\begin{abstract}
Carrying uncertain information via a multivalued function can be found in different settings, ranging from the computation of the image of a set by an inverse function to the Dempsterian transfer of a probabilistic space by a multivalued function. We then get upper and lower images. In each case one handles socalled 'thick sets' in the sense of Jaulin, i.e., lower and upper bounded ill-known sets. Such ill-known sets can be found under different names in the literature, e.g., "interval sets" after Y. Y. Yao, "twofold fuzzy sets" in the sense of Dubois and Prade, or "interval-valued fuzzy sets", ... Various operations can then be defined on these sets, then understood in a disjunctive manner (epistemic uncertainty), rather than conjunctively. The intended purpose of this note is to propose a unified view of these formalisms in the setting of possibility theory, which should enable us to provide graded extensions to some of the considered calculi.
\end{abstract}

Keywords: Thick set, interval analysis, possibility theory, inverse image, uncertainty, thick set, interval analysis, possibility theory, inverse image, uncertainty

\section{Introduction}

The links between interval calculus [19] and possibility theory [11,30] are well-known, as well as the interest for interval calculus in robust control [18]. The need for guaranteed approximation has led B. Desrochers and L. Jaulin to propose an original "thick" set and thick interval calculus [3,4]. This research note starts with the study of links of that latter calculus with other works dealing with uncertainty in the setting of possibility theory and fuzzy set theory.

\section{Thick sets and other related notions}

A thick set $[4][[\mathbb{A}]]$ on a referential $U$ (in general $\mathbb{R}^{n}$ ) is an interval in $2^{U}$ defined by a pair $\left(\mathbb{A}_{*}, \mathbb{A}^{*}\right)$ such that $\mathbb{A}_{*} \subset \mathbb{A}^{*}$, namely

$$
[[\mathbb{A}]]=\left[\mathbb{A}_{*}, \mathbb{A}^{*}\right]=\left\{\mathbb{A} \in 2^{U} \mid \mathbb{A}_{*} \subset \mathbb{A} \subset \mathbb{A}^{*}\right\}
$$


This means that it is an ill-known set that is lower and upper bounded. Formally speaking, it can be represented by a fuzzy set with a tri-valued membership function $\mu$ : $\mu: U \rightarrow\{0,1 / 2,1\}$ as, for instance, the "ensembles flous" in the sense of Gentilhomme [17] who represents concepts by means of central area $\mathbb{A}_{*}$ and a peripheral $\operatorname{area} \mathbb{A}^{*} \backslash \mathbb{A}_{*}$. We may also use sets based on Kleene logic, for which, in the peripheral area, relevant information for concluding to belonging or not is incomplete $(1 / 2$ means unknown). For instance, it is the case for rough sets [24] where uncertainty comes from a lack of attributes for describing a set of objects exactly, or for twofold sets [10] where uncertainty comes from a lack of information on the attribute values of objects. So for rough sets, their extension is known, but their intension is ill-known (due to the lack of a sufficient number of attributes for discriminating elements), while it is the converse for twofold fuzzy sets, their intension is known and their extension is ill-known (the lack of information on attribute values prevent to from deciding whether or not an element satisfies or not a prescribed set of properties). See also the case of "interval sets" [27, 28].

Such generalized sets, at least viewed as a nested pair of sets, have been also introduced in the fuzzy set literature at different times. Let us mention interval-valued fuzzy sets (Zadeh [29], Sambuc [25] ) in particular, which are thick sets in the sense of Jaulin, namely, pairs $\left(\mathbb{F}_{*}, \mathbb{F}^{*}\right)$ of fuzzy sets that bracket an ill-known fuzzy set $\mathbb{F}$ : $\mu_{\mathbb{F}_{*}} \leq \mu_{\mathbb{F}} \leq \mu_{\mathbb{F}^{*}}$ (a particular case of type 2 fuzzy sets [29]). There are also "twofold fuzzy sets" [10], which are pairs of fuzzy sets that are strongly nested (the support of the former is included in the core of the other). They represent a set of elements that belong more or less necessarily (certainly) to $\mathbb{F}$, itself included in a superset of elements that belong more or less possibly to $\mathbb{F}$, these two fuzzy sets being induced by the fact that the relevant information for concluding to belonging or not is incomplete. Besides, a fuzzy set maybe viewed as a representation of a set with an ill-known location between the core and the support of the fuzzy set, see, e.g., [23].

\section{Epistemic sets and ontic sets}

For making clear the intended meaning of thick sets, it is important to understand what represent the sets handled in their definition. A set, be it classical or fuzzy, may represent

- either a constituted entity viewed as the conjunction of its elements - we then speak of d'ontic sets, if nce the set either represents a real object, or constitutes the entity that we are trying to identify.

- or a set of mutually exclusive possible values for a variable - we then speak of epistemic sets if nce it reflects an imprecise piece of information on the value of a variable.

This distinction is crucial for the proper handling of sets in computations. Thus a thick set, as a set of sets, is epistemic and represents an ill-known set, which is itself considered as ontic; for example, a physical area for which one wants to guarantee the coverage [14], for instance for making sure that a robot can pass in between two obstacles $[15,16]$, is an ontic set. 


\section{Dempster's construction}

An example of thick interval is made by the pair of lower and upper sets of solutions, $A_{*}$ and $A^{*}$ respectively, of the set equation

$$
f(S)=A \subseteq \Omega
$$

where $A$ is set and $f: U \rightarrow \Omega$ is an ill-known function belonging to a set $\Gamma$ of functions. It is a thick set inversion problem the solutions $S$ of which all satisfy $S \in$ $\left[A_{*}, A^{*}\right]$, with

$$
\begin{aligned}
A_{*} & =\{u: \forall f \in \Gamma, \exists a \in A \mid a=f(u)\} \\
& =\{u: \Gamma(u) \subseteq A\}=\bigcap_{f \in \Gamma} f^{-1}(A) ; \\
A^{*} & =\{u: \exists f \in \Gamma, \exists a \in A \mid a=f(u)\} \\
& =\{u: \Gamma(u) \cap A \neq \varnothing\}=\bigcup_{f \in \Gamma} f^{-1}(A) .
\end{aligned}
$$

Dempster [1] uses this model for inducing lower and upper probabilities from probabilistic space $(\Omega, P)$ and a multivalued mapping $\Gamma: \Omega \rightarrow U$. This mapping represents the incomplete knowledge about an aleatory variable, i.e., a function $f$ that relates a sample space to an observation space $U$. The value $u=f(\omega)$ is a measurement of a feature of $\omega$. IF an aleatory experiment gives a result $\omega$, the corresponding observation is an ill-known value $u=f(\omega) \in \Gamma(u)$ if nce the measurement tool that should yield $u=f(\omega)$ is imperfect. We are facing an ill-observed aleatory variable.

So we do not know the precise value of the probability $P_{f}(A)=P\left(f^{-1}(A)\right)$ of the event $f(\omega) \in A$ on $\Omega$, but only an upper bound $P^{*}(A)=P(\{\omega: \Gamma(\omega) \cap A \neq \varnothing\})$ and a lower bound $P_{*}(A)=P(\{\omega: \Gamma(\omega) \subseteq A\})$. The same construction can be made starting from a possibilistic space [9].

In this model, we are thus using the description of the inverse image $f^{-1}(A)$ of an event $A \subset \Omega$ when the function $f$ is ill-known. It is given by the thick subset $\left[A_{*}, A^{*}\right]$ of $\Omega$. The interval $\left[P_{*}(A), P^{*}(A)\right]=\left[P\left(A_{*}\right), P\left(A^{*}\right)\right]$ is the "probability" of this thick subset, and represents the set of possible values of the probability $P\left(f^{-1}(A)\right)$ when $f \in \Gamma$.

\section{Case of interval arithmetics}

An illustration of what precedes is given by the pair of lower and upper sets of solutions $X_{*}$ and $X^{*}$ of the equation $x-u=v$ (and thus $x=u+v$ ) where $u \in M, v \in N$, $M, N$ being intervals.

One may interpret the equation $x-u=v$ in an uncertain context in two ways:

- Looking for the set

$X^{*}=\{x: \exists u \in M, v \in N$, such that $x=u+v\}$.

- or looking for the set

$X_{*}=\{x: \forall u \in M, \exists v \in N$, such that $x=u+v\}$.

These maximal and minimal sets are respectively given by two set addition operations: namely Minkowski's subtraction and addition defined respectively by

$$
\begin{aligned}
X^{*}=M \oplus N & =\{x:(x \ominus M) \cap N \neq \varnothing\} \\
& =\{u+v: u \in M, v \in N\} \\
X_{*}=M \boxplus N & =\{x:(x \ominus M) \subseteq N\}
\end{aligned}
$$


with $x \ominus M=\{x-u \mid u \in M\} . M \boxplus N$ is the largest subset $S$ such that $\forall x \in$ $S, \exists v \in N, x-v \in M$. In other words, it is the subset of $x$ such that $-M$ translated by $x$ is included in $N$.

For instance, if $M=\left[m, m^{\prime}\right]$ and $N=\left[n, n^{\prime}\right]$, we have $M \oplus N=\left[m+n, m^{\prime}+n^{\prime}\right]$, and $M \boxplus N=$

$\left[m+n^{\prime}, m^{\prime}+n\right]$ if $m+n^{\prime} \leq m^{\prime}+n$ and $M \boxplus N=\varnothing$ otherwise. It can be checked that $M \boxplus N \subseteq M \oplus N$, and that the length of $M \boxplus N$ is the length of $M$ reduced by the one of $N$.

The operation $\oplus$ is said to be optimistic, and the operation $\boxplus$ is said to be pessimistic. It can be checked that:

$$
\begin{aligned}
& M \boxplus N=\bigcap_{f \in \Gamma} f^{-1}(N)=\bigcap_{u \in M} u \oplus N, \\
& M \oplus N=\bigcup_{f \in \Gamma} f^{-1}(N)=\bigcup_{u \in M} u \oplus N,
\end{aligned}
$$

where $u \oplus N=\{u+v \mid v \in N\}$ and $x \ominus M$ plays the role of $\Gamma(x) . \Gamma(u)=$ $\{f(x) \mid \exists u, f(x)=x-u$ and $u \in M\}$ and then $f^{-1}(N)=\{u+v \mid u \in M\}=M \oplus v$. Thus this is a particular case of Dempster's construction where $\Omega$ is the domain of $X$, and $U$ is the domain of $v$. In the notations of the previous section, one should write $M \boxplus N=N_{*}$ and $M \oplus N=N^{*}$. But here we see that $M \boxplus N$ and $M \oplus N$ do not solve the same problem.

If $M$ and $N$ are epistemic sets representing ill-known values, $M \oplus N$ describes the uncertainty about $x$ induced by the one on $u$ and $v$. If $N$ represents a tolerance interval to be respected, $M \boxplus N$ describes the values of $x$ allowed for making sure that the uncertainty about $x-u$ remains bounded by $N$ in spite of the fluctuations due to the poor knowledge about $u$, described by $M$.

But, let us suppose that $M$ and $N$ are ontic, and represent the positions on the real line of two rods. Then the length of $M \oplus N$ is the one of the rod obtained by concatenation of $M$ and $N$. By contrast, $M \boxplus N$ is the set of points certainly covered by the $\operatorname{rod} M$ if it is translated by a length $v \in N$.

These two operations $\boxplus$ and $\oplus$ can be generalized when $M$ and $N$ are fuzzy intervals $[5,8,26]$.

\section{An example: the problem of the two goats}

Let us consider two goats, each one is attached to a stake by a rope the length of which is $10 \mathrm{~m}$. The position of the stakes, $\mathbf{m}_{i}$ for goat $i, i \in\{1,2\}$ is ill-known. We only know that

$$
\mathbf{m}_{1} \in\left[\mathbf{m}_{1}\right]=[0,1] \times[2,10] \text { and } \mathbf{m}_{2} \in\left[\mathbf{m}_{2}\right]=[10,16] \times[0,1] .
$$

The area grazed by goat $i$ is pervaded by uncertainty, due to the uncertainty on the positions of the stakes. This is represented by the thick set

$$
\left[[\mathbb{A}(i)]=\llbracket\left[\mathbb{A}_{*}(i), \mathbb{A}^{*}(i)\right]\right.
$$


with

$$
\mathbb{A}_{*}(i)=\left[\mathbf{m}_{i}\right] \text { 田 } \mathbb{D}, \mathbb{A}^{*}(i)=\left[\mathbf{m}_{i}\right] \oplus \mathbb{D},
$$

where $\mathbb{D}$ is the disc with centrer 0 and radius 10 . The area grazed by at least one goat is the set $\mathbb{A}$ that belongs to the thick set

$$
\left.\llbracket \mathbb{A}]=\llbracket \mathbb{A}(1)] \cup \llbracket \mathbb{A}(2)]=\llbracket \mathbb{A}_{*}(1) \cup \mathbb{A}_{*}(2), \mathbb{A}^{*}(1) \cup \mathbb{A}^{*}(2)\right] .
$$

The set $\mathbb{A}$ is an ontic set, while the rectangles $\left[\mathbf{m}_{1}\right],\left[\mathbf{m}_{2}\right]$ are epistemic (in black on the figure). We are certain that none of the goats can reach the area in dark grey. Let us note that the possible grazed areas are not all the subsets between $\mathbb{A}_{*}(1) \cup \mathbb{A}_{*}(2)$ and $\mathbb{A}^{*}(1) \cup \mathbb{A}^{*}(2)$. The thick set is an encompassing approximation of the grazed areas that are effectively possible.

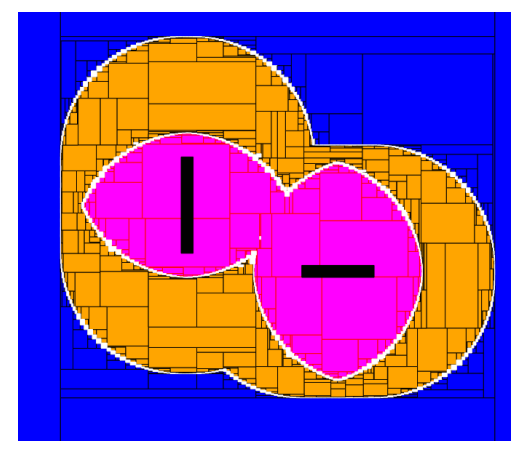

Fig. 1. The grazed area contains the set $\mathbb{A}_{*}$ (grey) and is contained in $\mathbb{A}^{*}$ (grey + light grey)

\section{Conclusion}

This note has been suggesting the existence of links between several works having different motivations. Thick sets are pairs of nested classical subsets. The framework of possibility theory should allow us to extend their calculus to the case of fuzzy thick sets, thus permitting to introduce gradedness in the uncertainty. However, for this fuzzy set extension, one may think of two approaches: i) working in terms of alpha level-cuts (which is certainly fine for the pessimistic part, but less obvious for the optimistic part), or ii) looking for the solution of a fuzzy set equation of the form $A+X=B$. This raises the question of the agreement between the two views. Besides, the work of Denœux et al. [2] can also be seen as an extension of thick sets to belief functions.

\section{Dedication}

This short note is dedicated to Hung T. Nguyen. The first and the last authors of this note had the privilege to meet Hung very early in the late seventies short after he published an 
important paper discussing the expression of Zadeh's extension principle (for extending a mapping to fuzzy arguments) in terms of alpha level-cuts with application to fuzzy arithmetics [20]. Hung has been a continuous supporter and contributor to fuzzy set theory, and the first and the last authors were fortunate enough to collaborate with him, in a friendly manner, on two overview papers on important fuzzy set issues [6] [7]. Among all his contributions, let us also particularly mention his pioneering works on the relation between belief functions and random sets [21], and his works on intervaland fuzzy-valued probabilities [22], a topic clearly related to the issues of this research note.

\section{References}

1. A. P. Dempster. Upper and lower probabilities induced by a multivalued mapping, Ann. Math. Statist., 38, 325-339,1967.

2. T. Denœux, Z. Younes and F. Abdallah. Representing uncertainty on set-valued variables using belief functions. Artificial Intelligence, Vol. 174, Issues 7-8, pages 479-499, 2010.

3. B. Desrochers, L. Jaulin, Computing a guaranteed approximation of the zone explored by a robot. IEEE Trans. Automat. Contr. 62(1): 425-430, 2017.

4. B. Desrochers, L. Jaulin, Thick set inversion. Artificial Intelligence 249: 1-18, 2017.

5. D. Dubois, H. Prade, Inverse operations for fuzzy numbers. Preproc. IFAC Symp. on Fuzzy Information, Knowledge Representation and Decision Analysis, Marseille, July 1921, 1983, 391-396. In: Fuzzy Information, Knowledge Representation and Decision Analysis (E. Sanchez, M.M. Gupta, eds.), Pergamon Press, 399-404, 1984.

6. D. Dubois, H. T. Nguyen, H. Prade, M. Sugeno. Introduction: the real contribution of fuzzy systems. In: Fuzzy Systems: Modelling and Control, (Hung T. Nguyen, M. Sugeno, eds.), Kluwer Academic Publ., Boston, 1-14, 1998.

7. D. Dubois, H. T. Nguyen, H. Prade. Possibility theory, probability and fuzzy sets: misunderstandings, bridges and gaps. In: Fundamentals of Fuzzy Sets, (D. Dubois, H. Prade, eds.), The Handbooks of Fuzzy Sets Series, Kluwer, 343-438, 2000.

8. D. Dubois, H. Prade, Fuzzy set-theoretic differences and inclusions and their use in the analysis of fuzzy equations. Control and Cybernetics (Warsaw), 13, 129-146, 1984.

9. D. Dubois, H. Prade. Evidence measures based on fuzzy information, Automatica, 21, 547562, 1985. Preliminary version: Upper and lower possibilities induced by a multivalued mapping. Preproc. IFAC Symp. on Fuzzy Information, Knowledge Representation and Decision Analysis, Marseille, Jul. 19-21, 1983, 174-152. In: Fuzzy Information, Knowledge Representation and Decision Analysis (E. Sanchez, ed.), Pergamon Press, 1984.

10. D. Dubois, H. Prade, Twofold fuzzy sets and rough sets - Some issues in knowledge representation. Fuzzy Sets and Syst., 23 (1), 3-18, 1987.

11. D. Dubois, H. Prade, Théorie des Possibilités. Masson, 1987.

12. D. Dubois, H. Prade, On incomplete conjunctive information, Computers and Mathematics with Applications, 15(10), 797-810, 1988.

13. D. Dubois, H. Prade, Gradualness, uncertainty and bipolarity: Making sense of fuzzy sets. Fuzzy Sets and Systems, 192, 3-24, 2012.

14. L. Jaulin, Solving set-valued constraint satisfaction problems. Computing, 94 (2-4), 297-311, 2012.

15. H. Farreny, H. Prade, Tackling uncertainty and imprecision in robotics. Proc. 3rd Inter. Symp. of Robotics Research, Gouvieux (Chantilly), (O. Faugeras, G. Giralt, eds.), M.I.T. Press, 8591, Oct. 1985. 
16. H. Farreny, H. Prade, Uncertainty handling and fuzzy logic control in navigation problems. Proc. Inter. Conf. on Intelligent Autonomous Systems, (L. O. Hertzberger, F.C.A. Groen, eds.), Amsterdam, Dec. 8-11, 1986, 218-225, North-Holland, 1987.

17. Y. Gentilhomme. Les ensembles flous en linguistique. Cahiers de Linguistique Théorique and Appliquée (Bucarest), 5, 47-63, 1968.

18. L. Jaulin, M. Kieffer, O. Didrit, E. Walter, Applied Interval Analysis: With Examples in Parameter and State Estimation, Robust Control and Robotics. Springer, 2001.

19. R. Moore, Interval Analysis. Prentice-Hall, 1966.

20. H. T. Nguyen. A note on the extension principle for fuzzy sets. J. Math. Anal. Appl., 64 (2) 369-380, 1978.

21. H. T. Nguyen. On random sets and belief functions. J. of Mathematical Analysis and Applications, 65 (3), 531-542, 1978. Reprinted in Classic Works of the Dempster-Shafer Theory of Belief Functions, (R. Yager, L. Liu, eds. ; A. P. Dempster, G. Shafer, advisory eds.), Studies in Fuzziness and Soft Computing, 219, chap. 5, 105-116, 2008.

22. H. T. Nguyen, V. Kreinovich, Berlin Wu, Gang Xiang. Computing Statistics under Interval anf Fuzzy Uncertainty. Applications to Computer Science and Engineering. Studies in Computational Intelligence 393, Springer, 2012.

23. H. T. Nguyen, V. Kreinovich, O. Kosheleva. Membership functions representing a number vs. representing a set: Proof of unique reconstruction. Proc. FUZZ-IEEE, 657-662, 2016.

24. Z. Pawlak. Rough Sets. Theoretical Aspects of Reasoning about Data. Kluwer Acad. Publ., Dordrecht, 1991.

25. R. Sambuc. Fonctions $\Phi$-floues. Application à l'aide au diagnostic en pathologie thyroidienne. Thèse Université de Marseille, 1975.

26. Solution of fuzzy equations with extended operations. Fuzzy Sets and Systems, 12, 237-248, 1984.

27. Y. Y. Yao. Interval sets and interval-set algebras. Proc. of the 8th IEEE Int. Conf. on Cognitive Informatics (ICCI'09), (G. Baciu, Y. Wang, Y. Yao, W. Kinsner, K. Chan, L. A. Zadeh, eds.), Hong Kong, June 15-17, 307-314, 2009.

28. J. T. Yao, Y. Yao, V. Kreinovich, P. Pinheiro da Silva, S. A. Starks, G. Xiang, H. T. Nguyen. Towards more adequate representation of uncertainty: From intervals to set intervals, with possible addition of probabilities and certainty degrees. Proc. FUZZ-IEEE, 983-990, 2008.

29. Quantitative fuzzy semantics. Information Sciences, 3, 159-176, 1971.

30. L. A. Zadeh. Fuzzy sets as a basis for a theory of possibility. Fuzzy Sets and Systems 1 (1978) 3-28. 